\title{
Lobelia rhynchopetalum und das Gewächshaus für tropische Hochgebirgs- pflanzen im Ökologisch-Botanischen Garten Bayreuth
}

\author{
Marianne Lauerer, Reiner Zimmermann, Lisa KirChner, Joseph Woodring \& Guido Arneth
}

\begin{abstract}
Lobelia rhynchopetalum, the Ethiopian Giant Rosette tree, is an endemic afro-alpine species of the Abyssinian high plateaus. Since 1994, it is cultivated in a specially designed greenhouse for tropical high elevation plants at the Ecological-Botanical Gardens of the University of Bayreuth. The first worldwide known flowering of this hapaxanthous giant rosette tree under greenhouse conditions in 1999 provided the opportunity to study its flowering biology under controlled conditions.

Lobelia rhynchopetalum flowers for about six months and produces a rapidly growing central inflorescence of several meters height which displays thousands of individual flowers. Each flower is proterandric and lasts for approximately three weeks. Large quantities of nectar is produced mainly during the male flowering phase. The nectar contains glucose and fructose in relatively low concentrations. Phases of high nectar production in Lobelia show concurrently a significantly elevated stem xylem sap flux with a pronounced maximum at midday. The results indicate that the pollinators of this species are day-active birds.
\end{abstract}

\section{Zusammenfassung}

Lobelia rhynchopetalum, der Äthiopische Schopfbaum, kommt endemisch in der afroalpinen Stufe des abessinischen Hochlandes vor und wächst seit 1994 auch im Ökologisch-Botanischen Garten der Universität Bayreuth in einem Spezialgewächshaus für tropische Hochgebirgspflanzen. Als dieser hapaxanthe Schopfbaum im Jahre 1999 weltweit erstmals in Kultur blühte, war dies Anlass, unter kontrollierten Bedingungen blütenbiologische Untersuchungen durchzuführen. Lobelia rhynchopetalum blüht etwa sechs Monate lang und bildet einen rasch wachsenden, mehrere Meter hohen Blütenstand mit tausenden von Einzelblüten. Die einzelne Blüte hat eine Blühdauer von etwa drei Wochen, ist vormännlich und produziert vor allem in der männlichen Blühphase große Mengen an Nektar, der in relativ geringer Gesamtkonzentration ausschließlich Glukose und Fruktose enthält. Die hohe Produktion an Nektar geht einher mit einem deutlich erhöhten Wasserfluss im Stamm der Lobelien, der ein ausgeprägtes Maximum zur Tagesmitte zeigt. Die vorliegenden Ergebnisse weisen darauf hin, dass tagaktive Vögel Bestäuber sind.

\section{Das Gewächshaus für Tropische Hochgebirgspflanzen}

Die alpine Stufe der tropischen Hochgebirge (über $3500 \mathrm{~m}$ ü. NN) ist durch extreme Umweltbedingungen und eine einzigartige Flora gekennzeichnet. Von entscheidendem Einfluss auf die Vegetation ist das sogenannte Frostwechselklima (Troll 1943). Die Temperaturen variieren im Tagesverlauf stärker (im Extremfall von $+20^{\circ} \mathrm{C}$ bis $-15^{\circ} \mathrm{C}$ ) als im Jahresverlauf; in der Nacht sinken sie regelmäßig und das ganze Jahr über unter den Gefrierpunkt, während sich die Bodenoberfläche tagsüber bis über $40^{\circ} \mathrm{C}$ erwärmen kann. HedBerG (1964) hat dieses Klima treffend mit „Winter jede Nacht, Sommer jeden Tag" beschrieben.

Botaniker der Universität Bayreuth, allen voran Prof. Dr. ERWIN BECK und seine Mitarbeiter, erforschen seit 1979 die Pflanzenwelt in den Hochlagen der ostafrikanischen Gebirge, insbesondere die Anpassungen der Pflanzen an die dort herrschenden Lebensbedingungen (z. B. BECK 1987, FETENE et al. 1998). Unter der Lei- tung des damaligen Direktors des ÖkologischBotanischen Gartens (ÖBG), Dr. GÜNTHER ROSSMANN, wurden aus ersten Ergebnissen dieser Untersuchungen und in Kooperation mit der TU München die Klima- und Bodenbedingungen für ein spezielles Hochgebirgshaus ÖBG erarbeitet, das 1994 in Betrieb genommen wurde. Mit Hilfe ausgetüftelter Technik gelingt es seit dieser Zeit, in dem relativ kleinen Gewächshaus (74 $\mathrm{m}^{2}$ Grundfläche, $50 \mathrm{~m}^{2}$ Pflanzfläche und $4 \mathrm{~m}$ Höhe), die klimatischen Bedingungen der $\mathrm{Na}-$ turstandorte $\mathrm{zu}$ simulieren und so die spezifischen Lebensformen und Pflanzenarten dieses Lebensraumes zu kultivieren. In dem Gewächshaus ist bei ganzjährig gleichbleibenden je zwölf Stunden Tag und Nacht ein täglicher Temperaturgang von $0^{\circ} \mathrm{C}$ in den Morgenstunden (6-7 Uhr) bis $20^{\circ} \mathrm{C}$ in der Mittagszeit (14 Uhr) eingestellt. Tiefere Temperaturen in der Nacht wären möglich, werden aber aus energetischen Gründen nicht realisiert. Das Gewächshaus ist zur thermischen Isolation von einem geschlossenen Raum mit eigener Regelung der Temperatur 
umgeben. Der 12-Stunden-Tag (8-20 Uhr) wird bei Bedarf (z. B. in den Wintermonaten zur Tagverlängerung) durch eine Zusatzbeleuchtung mit bis zu 52 Strahlern eingestellt. Verwendet werden 28 Hochdruck-MetallhalogendampfLampen à 1000 Watt und 24 HochdruckNatriumdampflampen à 400 Watt, die sich in einem temperaturgesteuerten und über eine Glasdecke vom Gewächshaus getrennten Lampenraum befinden.

Der täglich auftretende Frost bedeutet für Pflanzen am natürlichen Standort einen starken Stress. Da Eisbildung in lebenden Zellen zum Tod führt, müssen sich die Pflanzen gegen Frost schützen. Verschiedene Arten und Lebensformen erreichen dies durch unterschiedliche Strategien (BECK 1987). Die besondere Lebensform tropisch-alpiner Lebensräume sind Schopfbäume, deren Blätter an der Stammspitze angeordnet sind. Dadurch befinden sich die Blätter und das Spitzenmeristem der Pflanze so weit über dem Boden (außer in der Jugend, Abb. 4), dass sie aus dem Bereich der größten Temperaturamplitude und der stärksten Fröste herausgehoben sind. Im Gewächshaus des ÖBG werden Schopfbäume sowohl aus den südamerikanischen Paramos (Puya alpestris, Espeletia spp.) sowie vor allem aus den afrikanischen Hochgebirgen kultiviert (Senecio kilimanjari, Lobelia deckenii, Lobelia keniensis, Lobelia telekii und Lobelia rhynchopetalum). Neben den spektakulären Schopfbäumen sind Horstgräser, Polsterpflanzen (z. B. Helichrysum-Arten), Rosettenpflanzen (z. B. Haplocarpha rueppellii) und kleinblättrige Zwerggehölze (z. B. Alchemilla argyrophylla) in den afrikanischen Hochgebirgen weit verbreitet und wachsen auch im Hochgebirgshaus des ÖBG, um diesen speziellen Lebensraum möglichst naturnah darzustellen. Die Simulation der extremen Klimabedingungen und die hier kultivierten Pflanzen machen dieses Gewächshaus

Abb. 1 (oben): Äthiopische Schopfbäume (Lobelia rhynchopetalum) auf dem Sanetti Plateau in den Bale Mountains Äthiopiens.

Abb. 2 (unten): Blühende Äthiopische Schopfbäume in den Simien Mountains in Nordäthiopien.
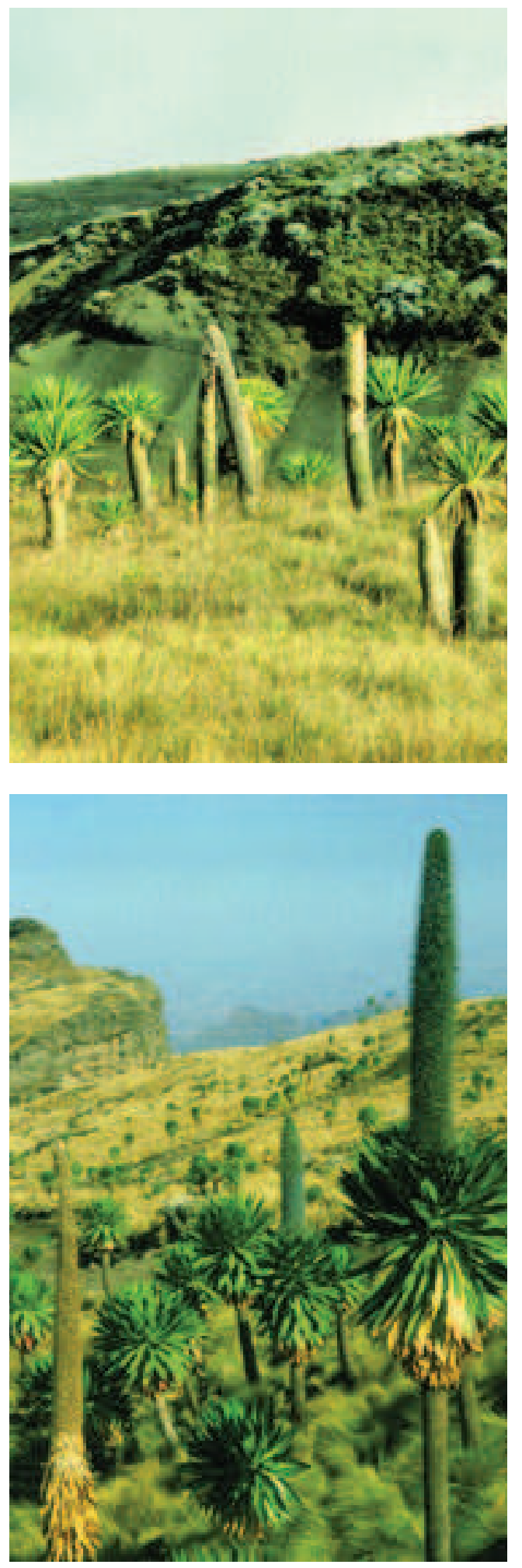

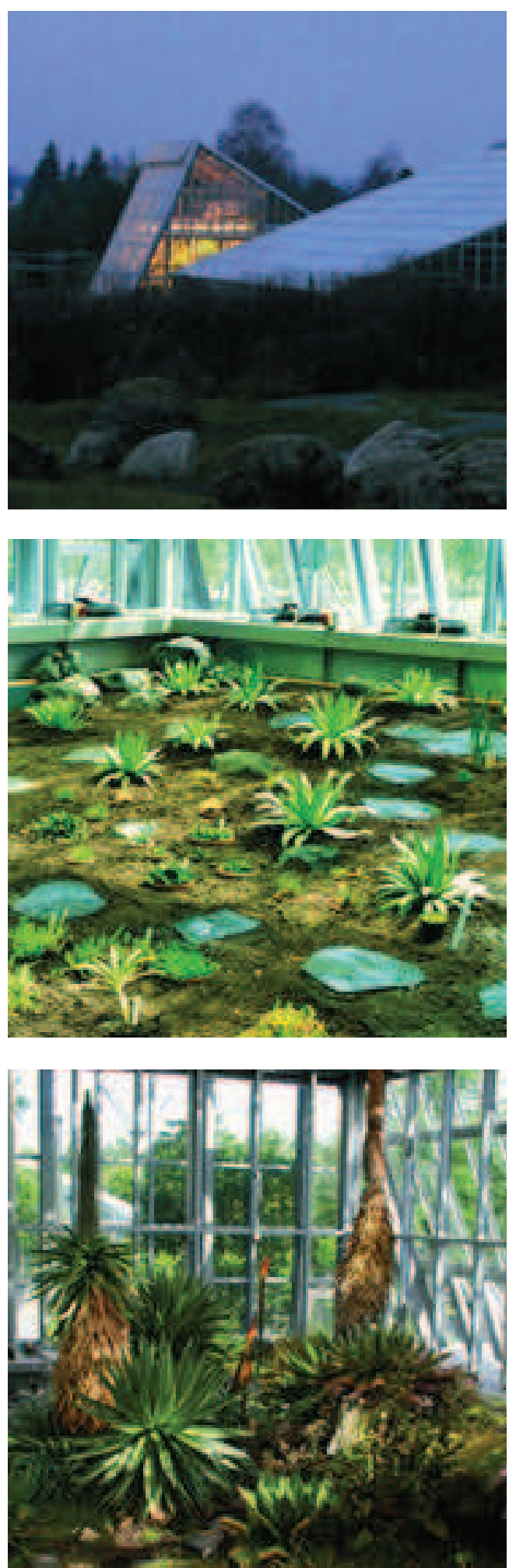

einzigartig. Es ist deshalb für Demonstrationszwecke und für die Forschung von großer Bedeutung.

\section{2. Äthiopischer Schopfbaum (Lobelia rhynchopetalum)}

\subsection{Biologie der Riesen-Lobelie}

Die Riesen- oder Baum-Lobelien (Lobelia L. sect. Rhynchopetalum (FRES.) BENTH \& HOOK. f., Lobeliaceae) gehören zu den eigenartigsten Pflanzen, die wir kennen (MABberley 1975). Sie kommen von Indien über die Inseln des nordwestlichen Pazifik bis Südamerika und Westafrika vor (MABberley 1975). Die größte Vielfalt zeigen diese pachycaulen Lobelien in den Hochgebirgen des östlichen Afrikas.

Eine für die Bergregionen Äthiopiens typische und dort endemische Art ist Lobelia rhynchopetalum HemsL. Dieser äthiopische Schopfbaum bildet in den Bale und Semien Mountains, wo er 1833 von EDUARD RÜPPELL entdeckt wurde (Rosen 1911), oberhalb der Waldgrenze (afroalpine Stufe, über etwa $3500 \mathrm{~m}$ ü. NN) ausgedehnte, aber lockere Bestände. Er wächst sowohl im relativ feuchten Nordabhang der Bale Mountains (dort etwa $800 \mathrm{~mm}$ Jahresniederschlag) wie in den trockeneren Simien Mountains im Norden Äthiopiens, wo eine drei- bis viermonatige Trockenzeit auftreten kann (NAUKE 1997). Der typische Habitus von L. rhynchopetalum ist der eines Schopfbaumes mit einem einzigen, drei bis sechs Meter hohen, unverzweigten Stamm und einem endständigen Blattschopf, der nach FETENE et al. (1998) aus 60-80 voll entwickelten und bis zu 100 und mehr jungen Blättern besteht, die die apikale Knospe umgeben. Eigenen

Abb. 3 (oben): Nächtlicher Blick auf das Gewächshaus für Tropische Hochgebirgspflanzen im Ökologisch-Botanischen Garten Bayreuth, Oktober 2003.

Abb. 4 (Mitte): Blick ins Gewächshaus für Tropische Hochgebirgspflanzen unmittelbar nach der ersten Anpflanzung u. a. mit Lobelia rhynchopetalum (1995).

Abb. 5 (unten): Blick ins Gewächshaus für Tropische Hochgebirgspflanzen im Juli 2009 (Blickwinkel ähnlich wie in Abb. 4) mit einer blühenden (links im Bild) und einer bereits verblühten (rechts hinten im Bild) Lobelia rhynchopetalum. 
Beobachtungen im Gewächshaus des ÖBG zufolge hatten die Lobelien $90 \pm 20$ voll entwickelte Blätter $(\mathrm{n}=6)$.

Lobelia rhynchopetalum entwickelt einen spektakulären, kolbenähnlichen Blütenstand, der sich aus dem apikalen Meristem entwickelt (Rosen 1911). Nach der Samenreife stirbt die ganze Pflanze ab (hapaxanth) oder regeneriert sich durch basale Seitensprosse (Adventivsprosse, BECK et al. 2002), von welchen wiederum einige zur Blüte kommen können. Die „Baumskelette” jedoch bleiben in der offenen Landschaft der afro-alpinen Zone mehrere Jahre stehen und geben ihr das charakteristische Gepräge.

Im Zuge von Forschungsaufenthalten Bayreuther Botaniker wurden 1992 Samen dieses Schopfbaumes in Äthiopien geerntet, im ÖBG ausgesät und seit 1994 im Spezialgewächshaus in Bayreuth kultiviert. Bereits im Winter 1999/2000 kam die erste Pflanze zur Blüte, was am Naturstandort erst im Alter von 15 oder mehr Jahren geschieht.

\section{3. „Die bombastische Blüte von Bayreuth“}

So lautete die Überschrift eines Presseberichtes, als der erste Äthiopische Schopfbaum im Gewächshaus für tropische Hochgebirgspflanzen zur Blüte kam - weltweit zum ersten Mal unter Kulturbedingungen. Am Naturstandort sind langfristige, ökophysiologische Messungen oft mit praktischen Schwierigkeiten verbunden. Die sieben in den folgenden Jahren unter standardisierten Bedingungen im Gewächshaus kultivierten und blühenden Lobelien boten ideale Möglichkeiten für die Analyse phänologischer und

Abb. 6 (oben): Der Blütenstand einzelner blühender Lobelien erreicht stattliche Höhen, in die Decke des Gewächshauses musste ein Loch geschnitten werden, um den Blütenstand aufrecht weiter wachsen lassen zu können (Februar 2005).

Abb. 7 (Mitte): Ausschnitt aus dem Blütenstand einer Lobelie.

Abb. 8 (unten): Wachstum der Blütenstände von vier Lobelia rhynchopetalum im Gewächshaus für Tropische Hochgebirgspflanzen des ÖBG, die zwischen 1999 und 2005 blühten. Die Messungen des täglichen Wachstums wurden bei einer Blütenstandslänge von $50 \mathrm{~cm}$ begonnen (Tage $=0$ ).
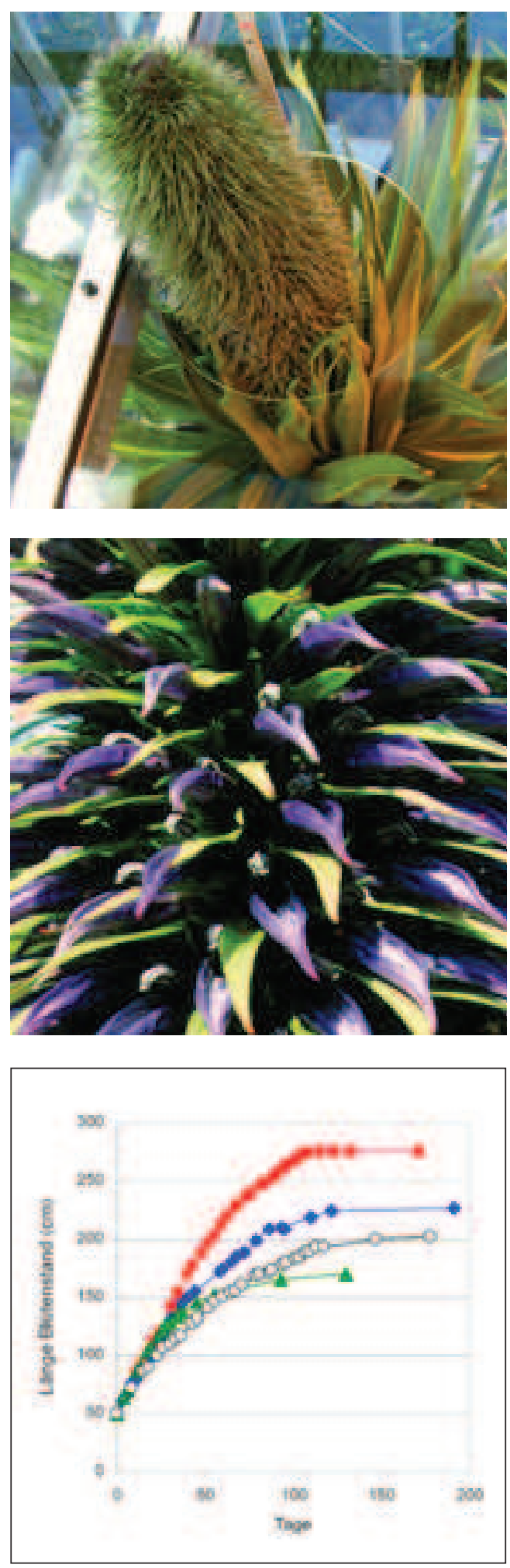

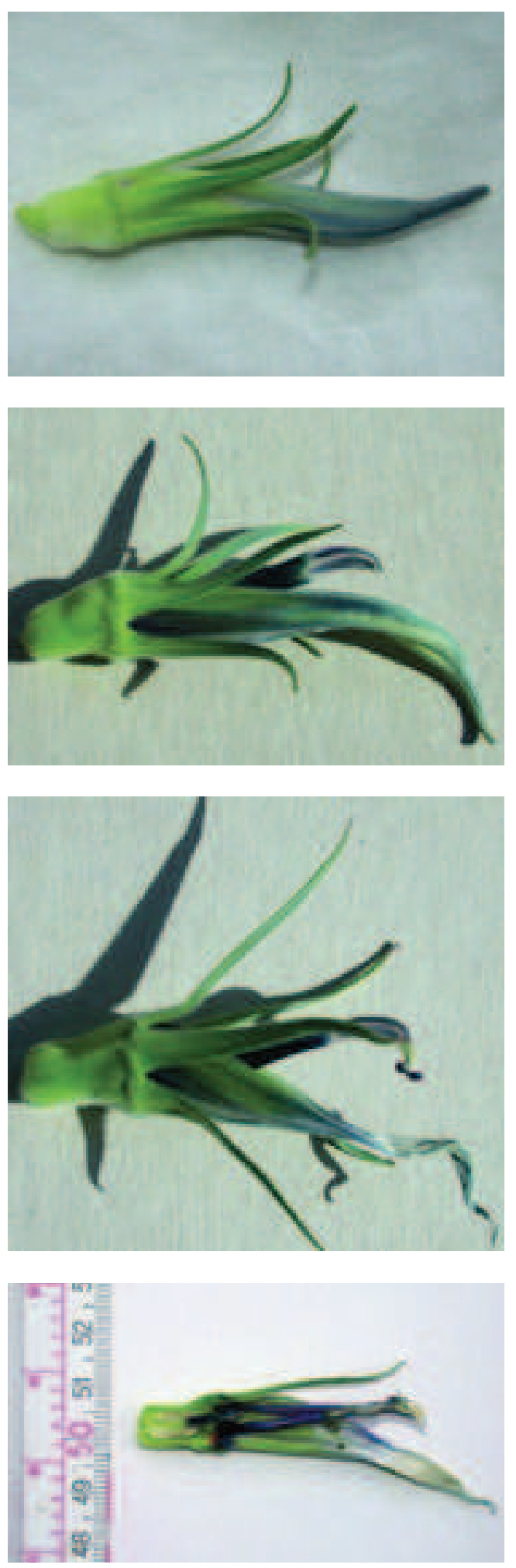

blütenbiologischer Parameter (Wachstum des Blütenstandes, Nektarproduktion und -zusammensetzung) sowie des Wasserverbrauchs nicht blühender und blühender Lobelien.

Der terminale, unverzweigte Blütenstand wird am Naturstandort bis zu $4 \mathrm{~m}$ hoch (NAUKE 1997, Rosen 1911) und kann mehrere tausend Einzelblüten tragen (MABBERLEY 1975). Im Gewächshaus des ÖBG erreichte er maximal eine Länge von 2,8 m mit insgesamt knapp 2000 Einzelblüten. Die mittlere Höhe des Blütenstandes betrug bei allen bisher vermessenen Individuen $2,2 \pm 0,4 \mathrm{~m}(\mathrm{n}=4)$. Die Blühdauer beträgt etwa fünf Monate, der Blütenstand wächst dabei pro Tag bis zu 3,5 cm in die Höhe. Die blass lila bis blauen Blüten sind spiralig an der Blütenstandsachse angeordnet, jede Blüte wird etwa 5-7 cm groß und hat eine Blühdauer von 20-30 Tagen. Der Kelch ist radiär und 5-teilig, die Blüten selbst sind zygomorph. Die fünf verwachsenen Petalen bilden eine Lippe, auf der der Nektar präsentiert wird. Die Antheren der fünf Staubblätter formen eine Röhre, durch die der Griffel wächst und dabei den Pollen apikal aus der Röhre hinausschiebt (HEYWOOD 1993). Erst wenn der Griffel nach etwa 20 Tagen die Antherenröhre durchwachsen hat, entfalten sich die Narben und die weibliche Phase der Blüte beginnt. Die Zahl der Samen, die nur 0,3-1 mm groß sind und sich nach Fremdbestäubung bilden, ist außerordentlich hoch. Nach NAUKE (1997) enthält eine Frucht etwa 2800 Samen, bei über 2000 Blüten pro Blütenstand produ-

Abb. 9: Entwicklung einer einzelnen Blüte von Lobelia rhynchopetalum. oben: junge Blüte, bei der die fünf verwachsenen Blütenblätter noch geschlossen sind. 2.v. oben: Blüte im männlichen Zustand. Die fünf verwachsenen Blütenblätter bilden eine Lippe, auf der der Nektar präsentiert wird; die Staubblätter sind zu einer Röhre verwachsen. 3. v. oben: Blüte im weiblichen Zustand. Der Griffel hat sich durch die Antherenröhre geschoben, die zweiklappige Narbe ragt aus der Röhre heraus.

Abb. 10 (unten): Längs aufgeschnittene Blüte in der männlichen Blühphase. Der Griffel wächst durch die Staubblattröhre und schiebt dabei den Pollen aus der apikalen Öffnung. Die Narbe ist noch nicht empfängnisbereit. Deutlich zu erkennen ist das rote Saftmal an der Basis der Lippe. 
ziert eine einzelne Pflanze demnach mehrere Millionen Samen. Im ÖBG wurden die Blüten von Hand bestäubt und setzten reichlich Samen an, aus denen mittlerweile neue Pflanzen angezogen werden konnten.

\subsection{Blütennektar vom Äthiopischen \\ Schopfbaum: viel und süß}

Lobelia rhynchopetalum produziert Nektar, um Bestäuber anzulocken. Die Menge an Nektar pro Einzelblüte ist dabei erstaunlich hoch und variiert mit dem Entwicklungszustand der Blüte. Der meiste Nektar, bis zu $250 \mu \mathrm{l}$ pro Tag und Blüte, wird gebildet, wenn sich die proterandrische Blüte gerade öffnet. Im weiteren Blühverlauf nimmt die Nektarproduktion kontinuierlich ab, während der weiblichen Blühphase wird nur noch wenig Nektar gebildet. Der Vergleich mit anderen Pflanzenarten zeigt, dass L. rhynchopetalum relativ viel Nektar produziert. Zucchiniblüten (Cucurbita pepo, Cucurbitaceae) haben etwa $130 \mu$ l pro Blüte (DMiTruK 2006), Aesculus pavia (Sapindaceae) z. B. nur 7,8 $\mu$ l (BURKE et al. 2000) und die Orchidee Elleanthus bifarius nur $0,53 \mu \mathrm{l}$ (DzIEDZIOCH 2001). Semiramisia speciosa (Ericaceae) hat mit $190 \mu \mathrm{l}$ ähnlich viel Nektar wie L. rhynchopetalum, der Kapokbaum (Ceiba pentandra, Malvaceae; DZIEDZIOCH 2001) mit 1,25 ml Nektar sowie die Fledermausbestäubte Bromelie Werauhia gladioliflora mit

Abb. 11 (oben): Nektarproduktion der Blüte in Abhängigkeit von deren Entwicklungszustand. Mit Beginn der männlichen Phase der Blüte $\left(\sigma^{\prime}\right)$ wird der meiste und während der weiblichen Phase der Blüte $(q=$ = Beginn der weiblichen Blühphase) nur noch wenig Nektar gebildet.

Abb. 12 (Mitte): Zuckerkonzentration (Glukose und Fruktose) im Blütennektar von Lobelia rhynchopetalum in Abhängigkeit vom Entwicklungszustand der Blüte. Dargestellt sind Mittelwert und Standardabweichung ( $\mathrm{n}=8$; an jeweils 4 Blüten von zwei zeitgleich blühenden Individuen wurde täglich der Nektar abgenommen und mittels HPLC analysiert). Kleinbuchstaben geben signifikante Unterschiede für $\mathrm{p}<0,05$ (Tukey HSD-Test) wieder.

Abb. 13 (unten): Tagesgang der Lufttemperatur in 0 und $1 \mathrm{~m}$ Höhe über dem Boden in den Bale Mountains (oben, aus FETENE et al. 1998) und im Hochgebirgsgewächshaus des ÖBG (unten).
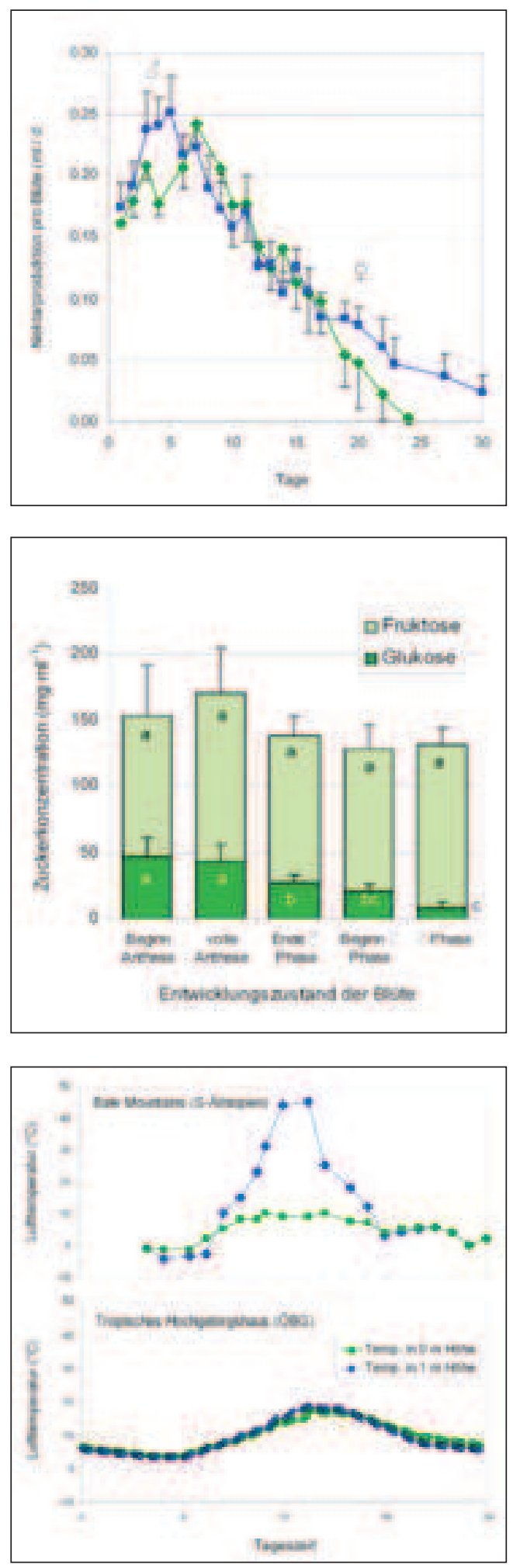

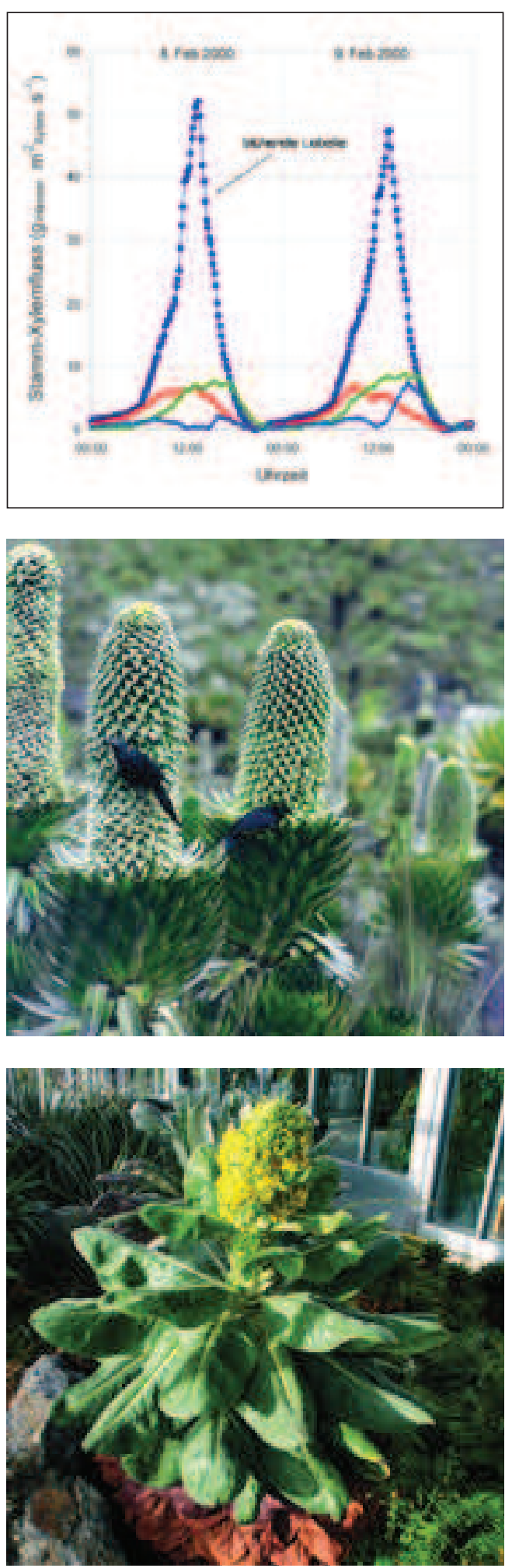

$1,1 \mathrm{ml}$ (TsChapka \& VON Helverseon 2007) produzieren deutlich mehr Nektar pro Blüte.

Die Konzentration an Zucker im Nektar von L. rhynchopetalum ist vergleichsweise gering und war über den gesamten Blühzeitraum mehr oder weniger konstant bei 130 bis $200 \mathrm{mg} / \mathrm{ml}$ (im Mittel $144 \mathrm{mg} / \mathrm{ml}$ ), was 13 bzw. 20 Gewichtsprozent Zucker entspricht. Trotz der geringen Konzentration ist die Gesamtmenge an Zucker im Nektar, die pro Blütenstand gebildet wird, erstaunlich hoch: Nimmt man pro Blütenstand 2000 Blüten an, von denen jede während der gesamten Blühdauer insgesamt 3,2 ml Nektar mit einer mittleren Zuckerkonzentration von $144 \mathrm{mg} / \mathrm{ml}$ produziert, so ergibt dies pro Blühereignis einer Pflanze mehr als 6 Liter Nektar und einen Gesamtzuckergehalt von $920 \mathrm{~g}$ !

Auffällig ist, dass der Nektar von L. rhynchopetalum keine Saccharose enthält, wie das sonst meist bei Blütennektar der Fall ist, sondern nur Glukose und Fruktose. Der Anteil an Fruktose ist dabei stets deutlich höher als derjenige an Glukose und das Verhältnis zwischen Fruktose und Glukose nimmt im Laufe der Blühphase zu, indem der Glukose-Gehalt von 50 auf $10 \mathrm{mg}$ pro ml sinkt, der Gehalt an Fruktose jedoch konstant bei etwa $130 \mathrm{mg}$ pro $\mathrm{ml}$ bleibt.

Die Ergebnisse der Nektaranalysen bei L. rhynchopetalum lassen auf eine Bestäubung durch Vögel (Nektarvögel) schließen, da bekannt ist, dass durch Vögel bestäubte Blüten viel Nektar produzieren, der jedoch eine Zuckerkonzentration von nur ca. $20 \%$ enthält (BAKER 1975, WiLSON et al. 2006). Dadurch ist der Nektar weniger viskos und kann so leichter von den mit einer Kapillarzunge ausgestatteten Vögeln aus

Abb. 14 (oben): Wasserfluss durch den Stamm einer blühenden Lobelie (Lobelia rhynchopetalum, dunkelblau) sowie drei nicht blühender Lobelien (rot, grün, blau) im Tagesverlauf. Die Messungen wurden im Hochgebirgshaus des Ökologisch-Botanischen Gartens durchgeführt und zeigen die Daten des 8. und 9. Februar 2000.

Abb. 15 (Mitte): Nektarvögel am Blütenstand von Lobelia keniensis, einem kleinen Schopfbaum, der endemisch am Mt. Kenya vorkommt.

Abb. 16 (unten): Blühender Senecio kilimanjari im Gewächshaus (Oktober 2008). 
der Blüte entnommen werden (DzIEDZIOCH 2001).

Interessant ist die Beobachtung, dass L. rhynchopetalum vorwiegend im männlichen Zustand der Blüte Nektar bildet und dadurch in diesem Zustand für Bestäuber attraktiver ist als in der weiblichen Phase. Dieses Phänomen ist vor allem bei zweihäusigen Arten bekannt und wird damit erklärt, dass die reproduktive Leistung einer männlichen Blüte mit der Anzahl der Blütenbesucher steigt, diejenige der weiblichen Blüten hingegen nicht von der Anzahl der Blütenbesucher abhängt (THOMSON et al. 1989, KlinKHAMER \& DE JONG 1990). Die weiblichen Blüten von L. rhynchopetalum locken die bestäubenden Vögel vermutlich durch die deutlich hervortretende purpurrote Narbe sowie das rote Saftmal an der Basis der Blüte an, das durch den Nektartropfen beim Blick in die Blüte optisch vergrößert erscheint.

\subsection{Literweiser Wasserdurchfluss}

Die hohe Nektarproduktion zeigt sich auch im Wasserverbrauch der Pflanze. Durch den Stamm einer blühenden Lobelie fließen pro Tag etwa drei Liter Wasser und damit - bei etwa gleicher Größe und Blattbiomasse - rund zehn Mal so viel wie bei einer nicht blühenden $(0,3$ bis 0,6 Liter). Vergleichende Messungen der Transpirationsrate der Blätter zeigten keinen Unterschied zwischen blühenden und nicht blühenden Lobelien. Der erhöhte Wasserverbrauch blühender Individuen kann somit auf den Wasserbedarf der Blüten und hier vor allem auf die hohe Nektarproduktion zurückgeführt werden. Die zeitliche Dynamik des Wassertransportes, - thermoelektrisch direkt gemessen im Wasser leitenden Xylemgewebe des Stammes - enthüllte eine weitere Besonderheit: Nicht blühende Lobelien zeigten kein einheitliches Muster im Tagesgang des Stamm-Saftflusses. Blühende Lobelien hingegen hatten ein ausgeprägtes Maximum bei Sonnenhöchststand zur Tagesmitte, relativ unabhängig von mikroklimatischen Faktoren (bereits vor der maximal erreichten Lufttemperatur und bei recht konstanter Luftfeuchte). Dies weist auf eine endogen bedingte Rhythmik im Wasserverbrauch blühender Lobe- lien hin und könnte als Anpassung an die Bestäubung durch Nektarvögel gedeutet werden, die am Naturstandort die Blüten bestäuben und vor allem zur Mittagszeit aktiv sind.

\section{Literatur}

BAKER, H. G. 1975: Sugar concentration in nectars from hummingbird flowers. Biotropica 7: 37-41.

BECK, E. 1987: Die Frostresistenz der tropisch-alpinen Schopfbäume. Naturwissenschaften 74: 355-361. BeCK, E., NAuke, P. \& Fetene, M. 2002: Hotspots der Biodiversitätsentwicklung. Tropische Hochgebirge. BIUZ 32: 82-88.

Burke, J. M., Wyatt, R., DE PAmphilis, C. W \& ARnold, M. L. 2000: Nectar characteristics of interspecific hybrids and their parents in Aesculus (Hippocastanaceae) and Iris (Iridaceae). J. Torrey Bot. Soc. 127: 200-206.

DMITRUK, M. 2006: Flowering biology, nectar production and insect visits in Cucurbita pepo L. flowers. - Acta Agrobotanica 59: 183-197.

DzieDZIOCH, C. 2001: Artenzusammensetzung und

Ressourcenangebot kolibribesuchter Pflanzen im Bergregenwald Südecuadors. - Dissertation Universität Ulm. Fetene, M., Gashaw, M., Nauke, P., Beck, E. 1998: Microclimate and ecophysiological significance of the tree-like life-form of Lobelia rhynchopetalum in a tropical alpine environment. Oecologia 113: 332-340.

HEDBERG, O. 1964: Features of afroalpine plant ecology. - Acta Phytogeographica Suecica 49: 1-144.

Hess, D. 1990: Die Blüte. - Stuttgart. HeYwood, V. H. (Hrsg.) 1993: Flowering plants of the world. - New York.

Klinkhamer, P., G., L \& DE JONG, T. J. 1990: Effects of plant size, plant density and sex differential nectar reward on pollinator visitation in the proteandrous Echium vulgare (Boraginaceae). - Oikos 57: 399-405.

Mabberley, D. J. 1975: The giant lobelias. Pachycauly, biogeography, ornithophily and continental drift. New Phytol. 74: 365-374.

NAUKE, P. 1997: The ethiopian Lobelia rhynchopetalum: Ecophysiology under natural and artificial conditions. Diplomarbeit Universität Bayreuth.

ROSEN, F. 1911: Die biologische Stellung der abessinischen Baumlobelie (Lobelia rhynchopetalum). - Beitr. biol. Pflanzen 10.

Thomson, J. D., Mcenna, M., A \& CruZan, M. B. 1989: Temporal patterns of nectar and pollen production in Aralia hispida: Implications for reproductive success. - Ecology 70 1061-1068.

Troll, C. 1943: Die Frostwechselhäufigkeit in den Luftund Bodenklimaten der Erde. Meteorologische Zeitschrift 60: $161-171$.

Wilson, P., Castellanos, M., C., Wolfe, A. D. \& ThomSON, J. D. 2006: Shifts between bee and bird pollination in Penstemons. - In: Waser, N. M. \& Ollerton, J. (Hrsg.): Plant-pollinator interactions. - Chicago.

Tschapka, M. \& von Helverseon, O. 2007: Phenology, nectar production and visitation behaviour of bats on the flowers of the bromeliad Werauhia gladioliflora in a Costa Rican lowland rain forest. - J. Trop. Ecol 23: 385-395. 